\title{
Low-Voltage CMOS Multiplier Circuit Based on the Translinear Principle
}

\author{
Behzad Ghanavati*, Enayatollah Taghavi Moghaddam \\ Sama Technical and Vocational Training College, Islamic Azad University, Mahshahr Branch, Mahshahr, Iran \\ *Corresponding Author: B.ghanavati@mahshahriau.ac.ir
}

Copyright (C) 2014 Horizon Research Publishing All rights reserved.

\begin{abstract}
In this paper, novel current-mode analog multiplier circuit based on a pair of voltage-translinear Loop is presented. The proposed circuit is designed in $0.18 \mu \mathrm{m}$ standard CMOS technology. Simulation result using HSPICE that verify the functionality of circuit with $1 \mathrm{~V}$ supply is presented. The circuit can find application in the implementation of wide range of analog systems, Fuzzy and Neural Network circuits.
\end{abstract}

Keywords Analog processing circuit, Translinear loop, Squarer Circuit, Low Voltage, and Multiplier

\section{Introduction}

Analog circuit design has received wide attention due to the supply voltage scaling down and their potential of lower power consumption [8, 10 from 12] and they are used in the fields of analog signal processing and parallel-computing neural or fuzzy systems. These applications usually require divider/multiplier circuits to cancel non-linearity or to implement modulators or aggregation operators [3-6].

Several techniques to design CMOS divider/multiplier circuits have been proposed in the literature.The first approach is to use the current-mode square root and squarer circuit based on the square-law characteristics of MOS transistors operated in the saturation region.[3] However, the power consumption by this approach is large; the square circuit requires bias currents for all input signals to make MOS transistors operate in the saturation region. The second approach is to use translinear principle of MOS transistor operated in the saturation or subthreshold region. [1,2]

The translinear principle, introduced by Barry Gilbert in 1975 [I], is one of the most important circuit theory contributions in the electronics era. In its original formulation, the translinear principle provides a simple and efficient way to analyze and synthesize nonlinear circuits based on bipolar junction transistors (BJTs). Due to their exponential characteristics, the translinear principle can be extended to MOS transistors operating in saturation. This approach has the advantage of low power consumption.

In this paper we proposed a new low-voltage divider/multiplier circuit based on the properties of translinear loop which is work in saturation region. The proposed circuit work with a single power supply $1 \mathrm{~V}$ which is lower than previous proposals and make it suitable for low-voltage and portable applications.

Section 2 reviews the properties of translinear loops. The proposed squarer circuit is described in section 3 . Section 4 presents the proposed low voltage divider/multiplier and simulation result is presented in section 5. Conclusion is presented in section 6 .

\section{Voltage Translinear Loops}

Consider, for instance, the circuits of Figs. $1 \mathrm{a}$ and $1 \mathrm{~b}$. They represent a four-transistor voltage-translinear loop in stacked and up-down topology, respectively, with two MOSFETs connected clockwise and another pair connected counterclockwise. Applying the KVL, the following expression is obtained for both cases:

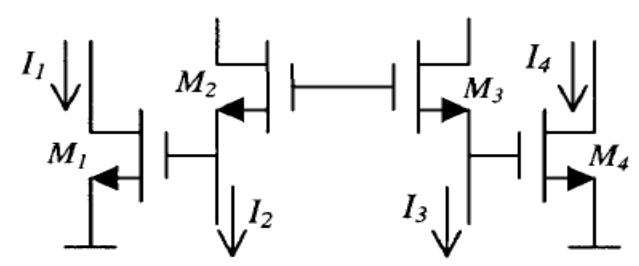

(a)

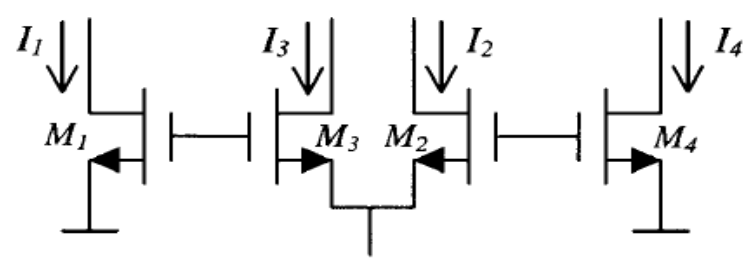

(b)

Figure 1. Voltage translinear-loop (a) Stacked Topology (b) Up-down topology 


$$
V_{G S 1}+V_{G S 2}=V_{G S 3}+V_{G S 4}
$$

And replacing $V_{G S}=V_{t h}+\sqrt{\frac{2 I}{\mu C_{o x} \frac{W}{L}}} \quad$ and assuming equal MOS transconductance factors and threshold voltages, the following expression is obtained:

$$
\sqrt{I_{1}}+\sqrt{I_{2}}=\sqrt{I_{3}}+\sqrt{I_{4}}
$$

Hence, several nonlinear current-mode functions can be implemented by properly injecting such currents. For instance, if we force

$$
I_{3}=I_{4}=\frac{I_{1}+I_{2}+2 I_{5}}{4}
$$

Being $I_{5}$ a certain current, after squaring both sides in (3) and rearranging, currents $I_{1} I_{2}$ and $I_{5}$ become related by

$$
I_{5}=\sqrt{I_{1} I_{2}}
$$

Therefore, a geometric-mean circuit is obtained if $I_{1}$ and $I_{2}$ are the input currents and the output current is a copy of $\mathrm{I}_{5}$.Alternatively, a squarer/divider is obtained if the output is a copy of $I_{2}$ and the inputs are $I_{1}$ and $I_{5}$. The simplest way to force (4) is to use the well-known structure of Fig. 2. However, the diode-connected MOSFET of the current mirror precludes very low voltage operation due to the stacking of two diode-connected transistors.

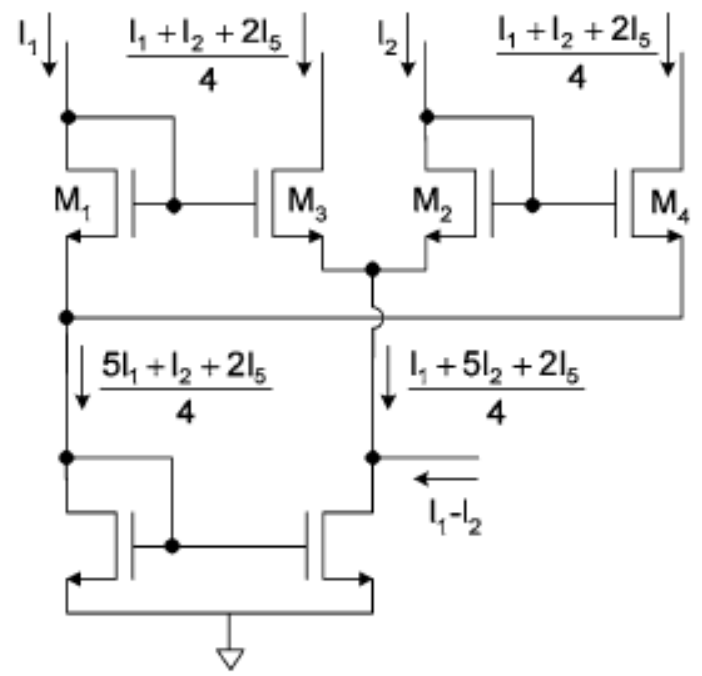

Figure 2. The square/Divider using stack loop

\section{The New Low-Voltage Squarer Circuit}

Equation (4) can be alternatively implemented using the novel topology of Fig. 3. Now a FVF, formed by transistors $\mathrm{M}_{1}$ and $\mathrm{M}_{\mathrm{F} 1}$, sets the proper reference dc voltage at the loop nodes. An advantage of this structure is that the source of $\mathrm{M}_{1}$ is a very low impedance node, so that voltage at this node is kept essentially constant regardless of the input and output current.

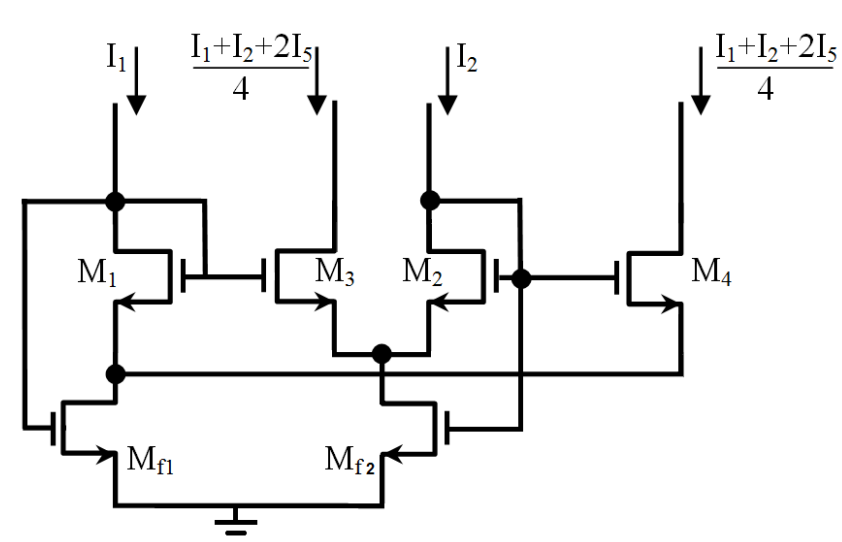

Figure 3. The proposed low-voltage squarer circuit

The minimum supply voltage is limited by the path formed by $\mathrm{I}_{\mathrm{b}}, \mathrm{M}_{1}$ and $\mathrm{M}_{\mathrm{F} 1}$ so the minimum supply voltage is

$$
V_{D D}^{\min }=V_{G S F}+2 V_{D S b s a t}
$$

where $\mathrm{V}_{\mathrm{GSF}}$ is gate-source voltage of $\mathrm{M}_{\mathrm{F} 2}, \mathrm{~V}_{\mathrm{DSbsat}}$ is the minimum voltage drop in current source $\mathrm{I}_{\mathrm{b}}$ and can be as small as $0.1 \mathrm{~V}$ in $0.18 \mu \mathrm{m}$ CMOS technology, $\mathrm{V}_{\text {th }}=0.55 \mathrm{~V}$ for NMOS so,

$$
V_{D D}^{\min }=V_{t h}+3 V_{D S b s a t}=0.55+3 * 0.1=0.85 \mathrm{~V}
$$

We have selected $\mathrm{V}_{\mathrm{DD}}=1 \mathrm{~V}$ in order to have an appreciable voltage swing.

\section{The New Low-Voltage Divider/Multiplier Circuit}

Following will described the principal of operation of the proposed Divider/Multiplier.

Consider the following expression:

$$
I_{\text {OUT }}=\frac{I_{1} I_{2}}{I_{3}}
$$

$\mathrm{I}_{1}, \mathrm{I}_{2}, \mathrm{I}_{3}$ and being current signals. Equation (6) can be equivalently written as

$$
I_{O U T}=\frac{\left(\sqrt{I_{1} I_{2}}\right)^{2}}{I_{3}}
$$

It becomes apparent that if we were able to calculate the geometric mean of two currents and then to introduce the output current into a squarer circuit having the following input-output characteristic:

$$
I_{\text {OUT }}=\frac{I_{1} I_{2}}{I_{3}}
$$

Then, according to (8), a current-mode multiplier/divider implementing could be obtained. This idea is shown schematically in Fig. 4, where the geometric-mean and squarer/divider outputs have been assumed to be flowing out. 


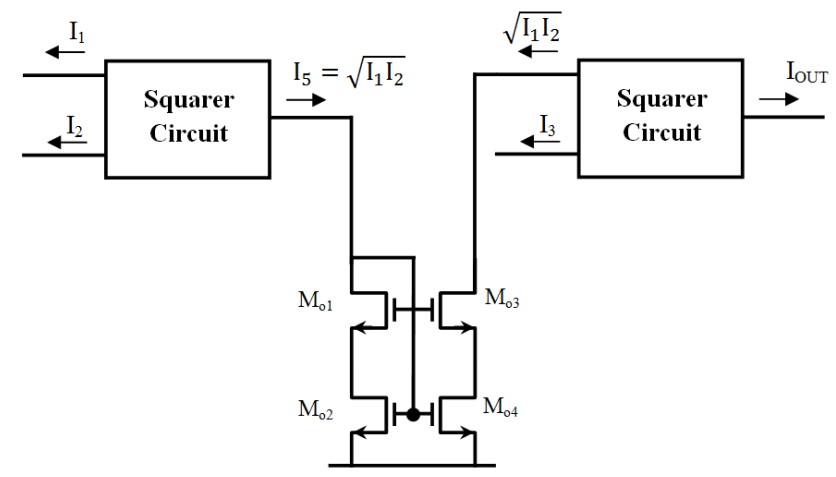

Figure 4. Principle of the multiplier/divider circuit

Fig.5 shows the proposed low-voltage current-mode divider/multiplier circuit. In this topology, $\mathrm{I}_{\mathrm{a}}$ is a constant bias current so the output current $\mathrm{I}_{\mathrm{OUT}}$ will be the multiplication of current $\mathrm{I}_{1}$ by current $\mathrm{I}_{3}$.

\section{Simulation Result}

The squarer circuit depicted in Fig. 3 and the current multiplier in Fig. 5 was laid out in standard $0.18 \mu \mathrm{m}$ CMOS technology. Post layout simulations from extracted circuit were performed for a $1 \mathrm{~V}$ single power supply voltage using HSPICE and level 49 parameters (BSIM3V3).

Fig. 6 shows SPICE simulation of the squarer circuit where a constant DC current is applied as $\mathrm{I}_{1}$ and a triangular current for $\mathrm{I}_{2}$. The result is showed by $\mathrm{I}_{\mathrm{OUT}}$.

The DC characteristic of proposed current multiplier is presented in Fig. 7 where a constant bias current is applied to the circuit as $\mathrm{I}_{3}$.

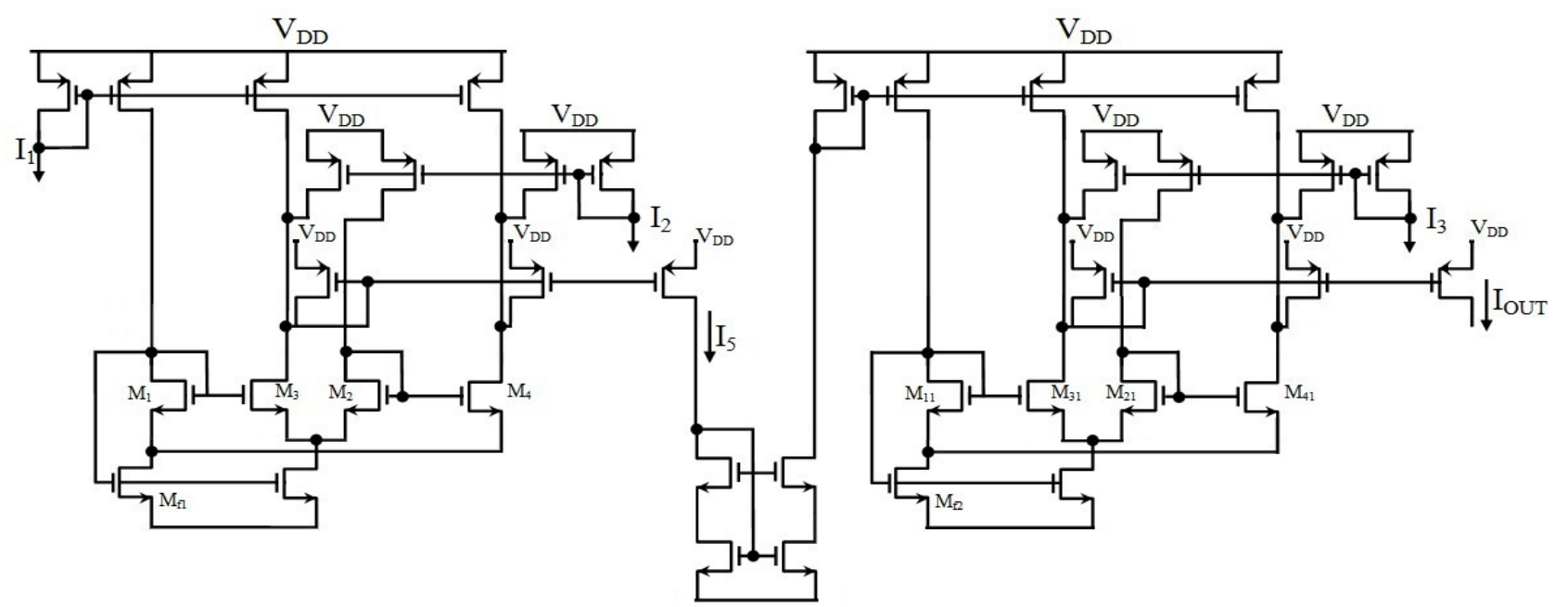

Figure 5. The low-voltage current divider

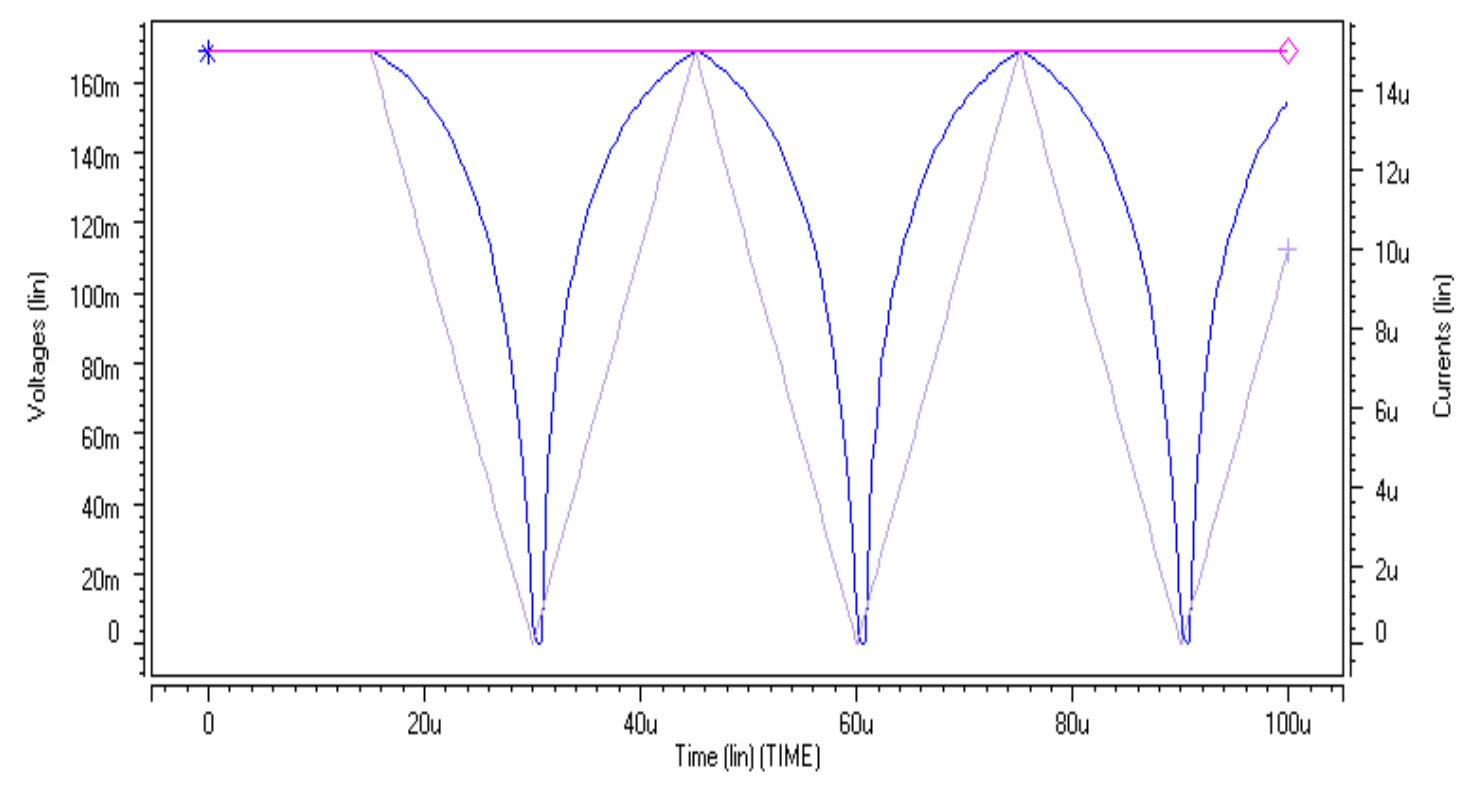

Figure 6. Simulation result of proposed squarer 
Table 1 summarizes some relevant result of the multiplier/Divider which proposed previously and compared them to this work.

Table 1. Summary of circuits performance and comparison

\begin{tabular}{|c|c|c|}
\hline Multiplier & Ref [11] & This work \\
\hline Technology & $2.4 \mu \mathrm{CMOS}$ & $0.18 \mu \mathrm{CMOS}$ \\
\hline Supply Voltage & $3.3 \mathrm{~V}$ & $1 \mathrm{~V}$ \\
\hline $\begin{array}{c}\text { Number of MOS TL } \\
\text { loop }\end{array}$ & 2 & 2 \\
\hline Power Consumption & $700 \mu \mathrm{W}$ & $90 \mu \mathrm{W}$ \\
\hline
\end{tabular}

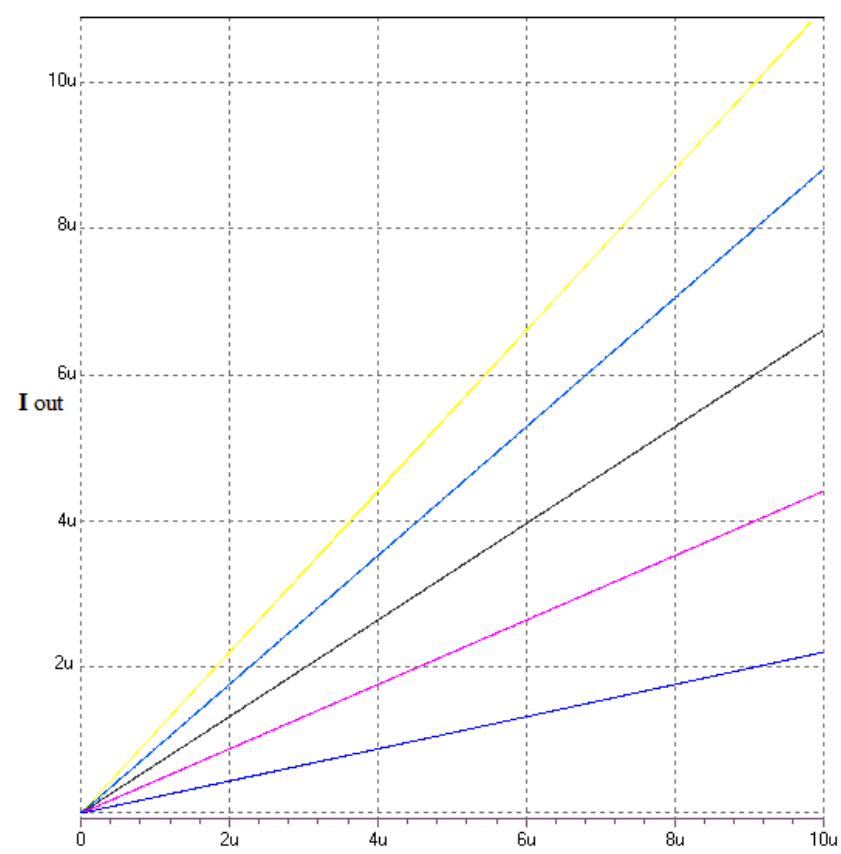

Figure 7. Simulated DC transfer characteristic of the multiplier $\left(\mathrm{I}_{\text {out }}\right.$ as a function of $\mathrm{I}_{1}$ with $\mathrm{I}_{3}=10 \mathrm{u}$ and $\mathrm{I}_{2}$ ranging from $2 \mathrm{uA}$ to $10 \mathrm{uA}$ in $2 \mathrm{uA}$ step)

\section{Conclusions}

A new, Low-voltage current Divider/Multiplier circuit based on properties of a translinear loop is described. The proposed circuit works with a single $1 \mathrm{~V}$ power supply which makes it suitable for designing low-voltage analog processing circuit.

\section{REFERENCES}

[1] A.Lopez-Martın, A. J. and Carlosena, A., "A systematic approachto the synthesis of square-root domain systems,"In Proc. of IEEEInt. Symp. on Circuits and Systems, Orlando, FL, vol. V, 1999,pp. 306-309.

[2] Liu, S. I., "Low voltage CMOS four-quadrant multiplier." Electron.Lett. 30(25), pp. 2125-2126, December 1994.

[3] Saxena, N. and Clark, J. J., "A four-quadrant CMOS analogmultiplier for analog neural networks." IEEE Journal of Solid- State Circuits 29(6), pp. 746-749, June 1994.

[4] Han, G. and Sanchez-Sinencio, E., "CMOS transconductance multipliers: A tutorial." IEEE Transactions on Circuits and System II 45(12), pp. 1550-1564, December 1998.

[5] Gai, W., Chen, H. and Seevinck, E., "Quadratic-translinear CMOS multiplier-divider circuit.” Electron. Lett. 33(10), pp. 860-861, May 1997.

[6] Gilbert, B., "Translinear circuits: A proposed classification." Electron. Lett. 11(1), pp. 14-16, January 1975.

[7] Seevinck, E. and Wiegerink, R. J., "Generalized translinear circuit principle." IEEE Journal of Solid-State Circuits 26(8), pp. 1098-1102, August 1991.

[8] Gilbert, B., "Translinear circuits: An historical overview." Analog Integrated Circuits and Signal Processing 9(2), pp. 95-118, March 1996.

[9] Eskiyerli, M. and Payne, A. J., "Square Root Domain filter design and performance." Analog Integrated Circuits and Signal Proc. 22, pp. 231-243, March 2000.

[10] Lopez-Martın, A. J. and Carlosena, A., "Geometric-mean based current-mode CMOS multiplier/divider." In Proc. of IEEE Int. Symp. on Circuits and Systems, Orlando, FL, vol. I, 1999,pp. 342-345.

[11] Lopez-Martın, A. J. and Carlosena, A.," Current-Mode Multiplier/Divider Circuits Based on the MOS Translinear Principle" Analog Integrated Circuits and Signal Processing, 28, 265-278, 2001 